\title{
An Effective Rule For Excavate Structural Co-Located Working Objects
}

\author{
Prof.Stefanie Backer, Sandra Kote
}

RWTH Aachen University Germany

\begin{abstract}
Mining co-location patterns from special databases might disclose the kinds of special options that area unit seemingly set as neighbors' in area. Consequently, we tend to gift associate degree rule antecedently for excavate specially co-located moving objects victimization spatial data processing techniques and Prim's rule. Within the previous technique, the scanning of information to mine the special co-location patterns took a lot of procedure price. So as to scale back the computation time, during this study, we tend to build use of R-tree that's special organization to mine the special co-location patterns. The vital step conferred within the approach is that the transformation of special information into the compact format that's wellsuitable to mine the patterns. Here, we've tailored the R-tree structure that converts the special information with the feature into the transactional formatting. Then, the distinguished pattern excavate rule, FP growth is employed to mine the special co-location patterns from the reborn format of information. Finally, the performance of the planned technique is compared with the previous technique in terms of your time and memory usage. From the results, we are able to make sure that the planned technique outperformed of concerning quite five hundredth of previous rule in time and memory usage.
\end{abstract}

Keywords: special data processing, Co-Location Patterns, Minimum Support, Minimum Bounding parallelogram, FP Tree, Vehicle Movement information

\section{Introduction}

An automated discovery of special information is needed attributable to the quick enlargement of special information and intensive use of special databases. Nowadays, the special data processing end up to be additional eminent and stimulating for the explanation that plentiful 
special information are hold on in special databases. Special data processing is outlined because the method of drawing out the attention-grabbing and antecedently unknown however in all probability relevant patterns from special databases. The excavate of pregnant patterns from special information sets is additional knotty than excavate the analogous patterns from conservative numeric and categorical data because of the problem of special information varieties, special relationships and special autocorrelation. In numerous applications, special patterns have excessive

Demand to work out the special co-location patterns in wide applications is that the main goal of special data processing. For each positive and negative association rules, special co-location and de-location patterns area unit identical. The subsets of mathematician special feature varieties area unit represented by special co-location patterns associate degreed its instances area unit typically positioned in shut geographic proximity special de-location patterns extend the conservative special associations so as to incorporate an association rules within the style of $A \rightarrow \neg B$, that denotes that $B$ won't exist near $A$.

\section{Literature Survey}

From special databases varied analysis works exist within the literature specialize in the topic of excavate special co-location patterns. Nowadays, the developing of ways for co-location pattern excavate has drawn a good concentration in world applications. During this topic, a number of the foremost recent literature works area unit depicted here. In giant special datasets the numerous co-location and de-location patterns are strip-mined employing a Co-location and Delocation patterns excavate rule (CODEM). Here, by suggests that of k-Nearest options (k-NF) the special close/separate relationships of co-location/de-location patterns in special datasets are analyzed. To make a decision the close/separation relationship between this feature and alternative options the k-NF set of 1 feature type's instances are used. After, by applying a correlation checking operation the irrelevant patterns are filtered.

Problem Description and Definition Given a collection of vehicles $V=, 1 \leq i \leq l$ wherever $I$ is that the most range of vehicles within the special. Every vehicle having worth the worth $\}$ of $x$ coordinate price and $y$ coordinate value $\mathrm{VI}=$. the whole special space is split into grids supported the worth of $P, G=x / p, y / p$ wherever $P$ is price that is use for divide the special space in $x$ coordinate and $y$ coordinate, here the grids area unit accustomed cowl the all the places in special space. 
Subsequently the special containing the amount of grids $G=$, wherever $1 \leq i \leq k$ here $k$ is that the total range of grids within the special. Every of the grid contain some range of vehicles if the amount of vehicles [n (VI) gi] is below minimum bounding parallelogram [n (VI) gi].

Problem Description and Definition Given a collection of vehicles $V=, 1 \leq i \leq I$ wherever $I$ is that the most range of vehicles within the abstraction. Every vehicle having worth the worth of $x$ coordinate worth and $y$ coordinate value $\mathrm{VI}=$. the entire abstraction space is split into grids supported the worth of $P, G=x / p, y / p$ wherever $P$ is worth that is use for divide the abstraction space in $x$ coordinate and $y$ coordinate, here the grids are wont to cowl the all the places in abstraction space. Subsequently the abstraction containing the quantity of grids $G=$, wherever $1 \leq i \leq k$ here $k$ is that the total range of grids within the abstraction.

\section{Algorithm for excavate Spatially Co-Located Moving Objects}

With the wide accessibility of GPS, wireless, telecommunication and internet technologies, large amounts of object movement knowledge are collected from varied moving object targets, like animals, mobile devices, vehicles and climate radars. Analyze such knowledge has deep implications in several applications, e.g., ecological study, control, mobile communication management and climatologically forecast. During this study, we tend to focus of our study on vehicle movement knowledge analysis and examine the excavate strategies for discovery of varied vehicle movement patterns. It's common that objects follow some regular movement patterns.

\section{Results and discussion}

The experimental results of the planned formula for abstraction co-located moving objects from the spatial databases are delineated here. The experimental results and analysis of the spatially co-located patterns of our planned formula are done here.

\section{Conclusion}

We have given Associate in nursing economical formula for excavate abstraction co-located moving objects that materializes spatial neighbor relationship and reduces the process price very with aid of the accepted FP Tree excavate formula. In 1st we have a tendency to split the realm by applying grids later on the minimum bounding parallelogram is employed to seek out teams 
of vehicles by elaborate the scale of the grid. Makes the vehicles list of every grid later on take away the de-located vehicles with the assistance of minimum support and named that list as changed vehicles list. FP tree made for the changed vehicles list later on the co-located vehicles are deep-mined from it. The spatially co-location excavate formula economical since the accepted frequent vehicle excavate is employed here. Finally, we've got generated the candidate co-location vehicles that satisfy the minimum bounding parallelogram

\section{References}

1. Al-Nay mat, G. Enumeration of top set for excavate abstraction co-location patterns. Proceedings of the IEEE/ACS International Conference on pc Systems and Applications.

2. Celia, M., J.M. Kang and S. Shear. Zonal colocation pattern discovery with dynamic parameters. Proceedings of the seventh IEEE International Conference on data processing.

3. Francis, F.S. and P. Thambidurai. Economical physical organization of R-trees victimization node cluster. J. Compute. Sci., 7: 206-214.

4. Sarnia, N.N. and M. Hemalatha. Estimation of evolutionary optimization algorithm for association rule using spatial data mining. 